\title{
Experimental Demonstration of a Suspended Dual Recycling Interferometer for Gravitational Wave Detection
}

\author{
G. Heinzel, ${ }^{1, *}$ K. A. Strain, ${ }^{2}$ J. Mizuno,,${ }^{1,}$ K. D. Skeldon, ${ }^{2}$ B. Willke, ${ }^{3}$ W. Winkler,${ }^{1}$ \\ R. Schilling, ${ }^{1}$ A. Rüdiger, ${ }^{1}$ and K. Danzmann ${ }^{1,3}$ \\ ${ }^{1}$ Max-Planck-Institut für Quantenoptik, Hans-Kopfermann-Strasse 1, D-85748 Garching, Germany \\ ${ }^{2}$ Department of Physics and Astronomy, University of Glasgow, Glasgow G12 8QQ, United Kingdom \\ ${ }^{3}$ Institut für Atom- und Molekülphysik, Universität Hannover, Callinstrasse 38, D-30167 Hannover, Germany
}

(Received 1 September 1998)

\begin{abstract}
The advanced scheme of "signal recycling" is to be used in the British-German GEO 600 project, in addition to "power recycling" (which has become standard for all laser-interferometer gravitational wave detector projects). This combination, "dual recycling," has been demonstrated for the first time on a fully suspended interferometer, the Garching prototype with $30 \mathrm{~m}$ arm length. Signal enhancement and power buildup were as predicted; operation was reliable, and a significant contrast enhancement was observed. A control system that can be extended to full scale gravitational wave detectors was employed. [S0031-9007(98)07923-X]
\end{abstract}

PACS numbers: 04.80.Nn, 07.60.Ly, 95.55.Ym

One of the most exciting developments in science in the coming decade is expected to be the direct observation of gravitational waves from sources such as supernovae, coalescing neutron stars or black holes, pulsars, or even the big bang (see Ref. [1] for a review). There are currently five projects [2-6] constructing gravitational wave observatories that employ laser interferometry. Of these, GEO 600 will be a forerunner in employing the novel technique of "dual recycling," with several of the others considering this technique for future stages of their projects.

Laser-interferometric gravitational wave detectors work by sensing the differential phase shift imposed on the light in the two (orthogonal) arms of an interferometer by a passing gravitational wave. This phase shift is converted into an observable light-power change at the output of the interferometer. When other noise sources have been sufficiently reduced, the smallest detectable signal is limited by the variance of the photon-counting statistics (shot noise). The shot-noise limited sensitivity is improved if the light energy (number of photons) stored in the interferometer can be increased.

A sketch of the optical layout of the dual recycling experiment is shown in Fig. 1. Mirrors for power recycling and signal recycling, denoted by $\mathrm{M}_{\mathrm{PR}}$ and $\mathrm{M}_{\mathrm{SR}}$, are added to the basic Michelson interferometer formed by mirrors $\mathrm{M}_{1}, \mathrm{M}_{2}$, and a beam splitter (BS). Power recycling [7,8] enhances the circulating light power and hence improves the signal-to-noise ratio. The interferometer is operated so that its output is held at an interference minimum and the power recycling mirror $\mathrm{M}_{\mathrm{PR}}$ is chosen and positioned to maximize the light amplitude in the "power recycling cavity," formed by the Michelson interferometer and $\mathrm{M}_{\mathrm{PR}}$.

The performance can also be improved by optimizing the time of interaction of the light with the gravitational wave (i.e., by choosing the optimal detector bandwidth). This can be realized by using either resonant (Fabry-
Perot) cavities or nonresonant optical delay lines in the arms of the interferometer. The Fabry-Perot system needs additional control loops to keep the arm cavities resonant, while the delay-line scheme is limited by the large mirror size required and by stray light problems.

If signal recycling [9] is incorporated into the design of the interferometer, however, the detector bandwidth can in principle be made as narrow as desired without the use of delay lines or arm cavities. Furthermore, signal recycling is a flexible and efficient way to enhance the sensitivity of an interferometer within a restricted bandwidth about an arbitrary user-selectable center frequency $[9,10]$. This is useful in searching for continuous radiation from known narrow band sources such as pulsars.

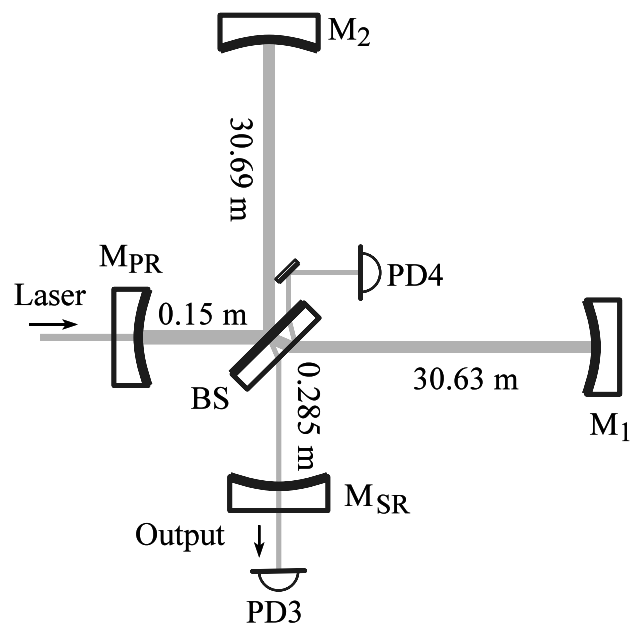

FIG. 1. The optical layout of the dual recycling interferometer. The power recycling mirror $\mathrm{M}_{\mathrm{PR}}$ and signal recycling mirror $\mathrm{M}_{\mathrm{SR}}$ are shown. The distance of each mirror from the center of the beam splitter (BS) surface in our experiment is marked. The power buildup in the power recycling cavity is indicated by the heavy gray lines. 
The signal sidebands produced by a passing gravitational wave will be separated from the carrier at the beam splitter and directed towards the signal recycling mirror $\mathrm{M}_{\mathrm{SR}}$. The amplitudes of these sidebands are enhanced when they are resonant in the "signal recycling cavity" formed by the Michelson interferometer and $\mathrm{M}_{\mathrm{SR}}$, leading to an equivalent enhancement of the light emerging through $\mathrm{M}_{\mathrm{SR}}$ and detected by PD3.

Dual recycling was first demonstrated on a small, benchtop interferometer [11] with rigidly mounted mirrors. However, full-scale detectors will use optical components that are suspended as pendulums to isolate them from seismic vibration. We report here on the construction and operation of a fully suspended dual recycled interferometer, carried out at the Garching $30 \mathrm{~m}$ prototype as part of the GEO 600 project.

To minimize unwanted motion and effects of refractive index fluctuations of the air, the whole interferometer system is enclosed in a vacuum chamber. For seismic isolation, the mirrors and the beam splitter are individually suspended as pendulums. Each component is actively damped with respect to local references. In addition, the relative positions and orientations of the mirrors are controlled to maintain the desired operating condition. The longitudinal control is described in this Letter, and the autoalignment system for the angular degrees of freedom will be the subject of a forthcoming paper [12].

The longitudinal degrees of freedom are the power recycling cavity length, the signal recycling cavity length, and the differential length of the two arms of the Michelson interferometer. In practice, these lengths are measured relative to the wavelength of the light from the source (an argon ion laser in our experiment). Thus the control of 1 degree of freedom, the power recycling cavity length in our experiment, can be replaced by the control of the laser frequency.

The signal for the power recycling and laser frequency control was obtained using the Pound-Drever-Hall method [13] with the phase modulation frequency chosen to be $12 \mathrm{MHz}$ (see Fig. 2). An additional prestabilization cavity was employed [14] which makes acquisition of lock simpler. At Fourier frequencies within the intended measurement band the laser frequency was locked to the power recycling cavity length. At frequencies below the measurement band the laser frequency and the power recycling cavity length were locked to the (relatively stable) length of the rigid reference cavity.

The signals for the remaining two longitudinal control loops were obtained by a variation of the method initially proposed by Schnupp [15]. Phase modulation was applied to the laser light before it enters the interferometer. With the parameters used in our $30 \mathrm{~m}$ experiment (and also those in GEO 600), the coupled cavity has a series of resonances separated approximately by the free spectral ranges of the power and signal recycling cavities (both near 4.85 MHz). The second such resonance, near $9.7 \mathrm{MHz}$, was chosen as the modulation frequency $f_{\mathrm{m}}$ in our experiment. An inten-

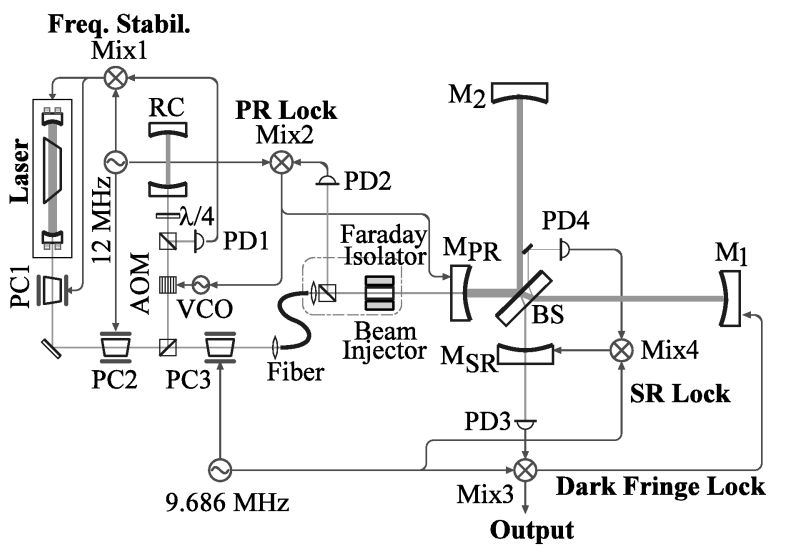

FIG. 2. Simplified diagram of the longitudinal control system for the dual recycling experiment. Light from the laser is phase modulated at $12 \mathrm{MHz}$, before a fraction is diverted to the reference cavity, RC. The Schnupp modulation $(9.7 \mathrm{MHz})$ is then applied before the light is directed to the interferometer through a single mode optical fiber. PC represents a Pockels cell, PD a photodiode, Mix an electronic mixer, VCO a $200 \mathrm{MHz}$ voltage-controlled oscillator, and AOM an acoustooptic modulator.

tional asymmetry in the arm lengths of the Michelson interferometer causes a fraction of the modulation sidebands to appear at the output port, even when the Michelson is at an interference minimum.

Any deviation of the Michelson phase from the dark fringe condition will then produce a proportional component at $f_{\mathrm{m}}$ in the photocurrent detected by PD3. The Michelson error signal is obtained by coherently demodulating this photocurrent and is also used to extract the gravitational wave information from the system.

The signal required to control the position of $\mathrm{M}_{\mathrm{SR}}$ was derived by a novel method employing the same Schnupp modulation as used for the dark-fringe lock, first tested in tabletop experiments without power recycling [16,17]. There is some sideband light incident on $\mathrm{M}_{\mathrm{SR}}$ and reflected back into the arms of the interferometer at all times. Any displacement of $\mathrm{M}_{\mathrm{SR}}$ will then change the phase of the sideband field in the arms of the interferometer with respect to the carrier field (which does not reach $\mathrm{M}_{\mathrm{SR}}$ in the desired operating condition). It can be shown that this provides a suitable locking signal for dual recycling with peak response at low frequency.

In our experiment (see Fig. 2), this signal is acquired by splitting off a small fraction $(\sim 0.1 \%)$ of the light in one arm at the rear surface of BS and directing it to PD4. The photocurrent from PD4 was demodulated coherently with the $9.7 \mathrm{MHz}$ modulation, using an appropriate demodulation phase.

Each of the signals used to control the interferometer was taken from the relevant demodulator, suitably amplified and filtered, and fed back to control the longitudinal positions of the 4 mirrors and the laser frequency. Feedback to the mirrors was accomplished via coil-magnet actuators that apply forces directly to the mirrors. 
The complexity of this asymmetrical optical system makes it difficult to predict its behavior intuitively. Thus, a method of simulating the error signals was developed [18] and used to design our experiment.

While a full description of the control system is beyond the scope of a Letter, a few key points are emphasized. The "unconditional" locking of the laser light frequency to the length of the power recycling cavity was much simplified by a slight asymmetry of the beam splitter's reflectance to transmittance $(51.5: 48.5)$ [8]. This has no significant effect on the signal at the output port, but ensures that there is always some light reflected back from the beam splitter towards the input port. This allows the power recycling cavity to be resonant with the incident light for a broad range of conditions of the interferometer, which was essential for lock acquisition of the dual recycling system.

It was found that, at least during acquisition of lock, the gain and bandwidth of the loop controlling $\mathrm{M}_{\mathrm{SR}}$ should be greater than that of the control loop for the Michelson dark fringe. In this condition the system normally settled to the desired operating state within a few seconds, and remained locked for several tens of minutes.

Tests were carried out to ensure that dual recycling was operating correctly with maximum response close to zero frequency. Power recycling was confirmed by monitoring the light power in the region of the beam splitter using calibrated photodetectors that sense a small fraction of the light inside the power recycling cavity, and also a fraction of the illuminating light from the laser. The observed power enhancement of $50 \pm 5$ is just what is expected with a 7\% transmittance power recycling mirror.

Coil-magnet actuators on $\mathrm{M}_{2}$ were used to apply to that mirror a sinusoidal longitudinal position modulation of $8.8 \times 10^{-13} \mathrm{~m}(\mathrm{rms})$ at $375 \mathrm{~Hz}$. The signal thus produced at the output (visible on the spectrum of the output signal shown in Fig. 3) was measured with dual recycling in operation. Subsequently the signal recycling mirror was misaligned (by $\sim 3 \mathrm{mrad}$ ) so that it was acting as a simple attenuator. Then the signal was seen to be 1800 times

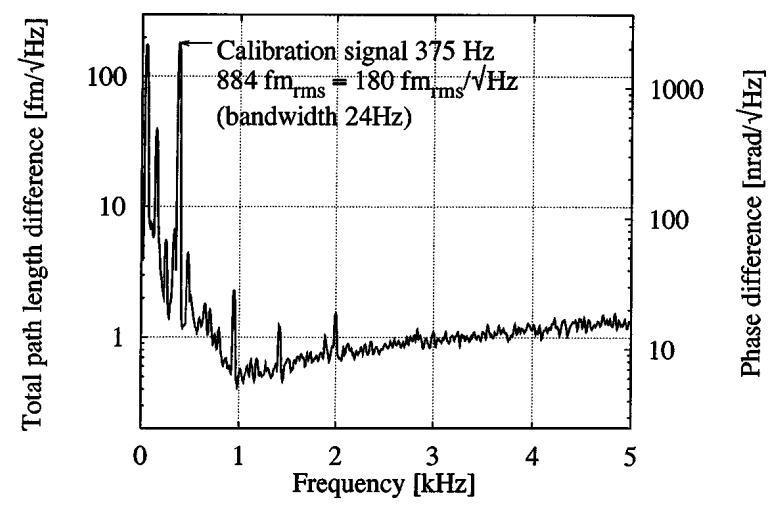

FIG. 3. A power spectrum of the typical output from the dual recycling system. The noise is technical in nature originating mainly from insufficiently suppressed laser frequency and amplitude fluctuations. smaller than before. Taking into account the $(3.9 \pm 0.2) \%$ transmittance of $\mathrm{M}_{\mathrm{SR}}$, the corrected enhancement was $70 \pm 4$.

This value is in reasonable agreement with the theoretically expected value of $91 \pm 5$, derived assuming lossless optical components. The enhancement consists of two factors: a signal recycling gain of $10.1 \pm 0.5$ (corresponding to the transmittance of $3.9 \%$ of the signal recycling mirror $\mathrm{M}_{\mathrm{SR}}$ ) and an improved coupling of Schnupp modulation sidebands to the output that should increase the detected signal by a factor of 9. (The parameters of the system were chosen to maximize the transfer of the modulation sidebands to the main photodetector with dual recycling working.) This agreement is possible only with correctly working dual recycling, and the shortfall is probably due to the losses at the optical components and by imperfect alignment of the signal recycling cavity.

An important feature of dual recycling is the mode cleaning effect. The "dark fringe" of an interferometer is in practice not completely dark, due to higher order transverse modes produced by imperfect optics and due to the modulation sidebands, which are necessary for phase detection. In order to reach the ideal shot-noise limited sensitivity, the modulation sidebands must dominate the light power at the photodetector [19].

By "closing" the output with the signal recycling mirror the whole interferometer becomes a (complex) resonant cavity. If the mirror curvatures are chosen to ensure that the predominantly excited higher order modes are nondegenerate with the fundamental mode, their amplitudes are strongly suppressed both inside the system and at the output port. This mode cleaning effect reduces the "waste" light and therefore allows a closer approach to the ideal shot-noise limited performance. The mode cleaning effect was clearly observed in our experiment.

A charge-coupled device (CCD) camera was used to record the shape of the emerging light beam with and without signal recycling. The addition of the signal recycling mirror suppressed most of the structure from the light at the output port, as shown in Fig. 4. It was also observed that the influence of intentional misalignment of the Michelson interferometer was considerably smaller with dual recycling. While manual alignment of the interferometer was very difficult (we relied on the automatic system), the dual recycling system was tolerant of a larger degree of intentional misalignment before any degradation in performance was seen.

For a more quantitative description of the mode-cleaning effect, we use the term "contrast" to represent the fraction of the light power leaking out towards photodetector PD3 relative to that incident on the beam splitter from the input side. The conventional concept of fringe contrast cannot readily be applied to a power-recycled system, where a true "bright fringe" never exists. Note that with this definition the contrast could be made perfect with an opaque mirror $\mathrm{M}_{\mathrm{SR}}$, and thus an increased contrast is useful only when at the same time the signal is enhanced, as is the case in 


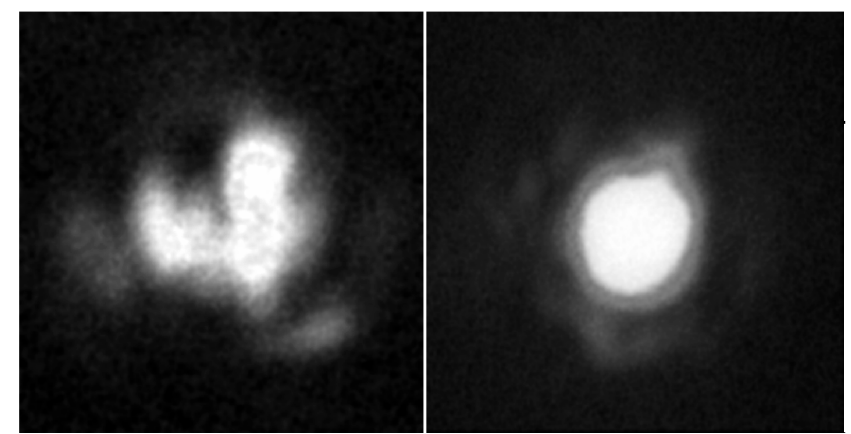

FIG. 4. CCD images of the output light from the $30 \mathrm{~m}$ interferometer. The left image shows the best result with power recycling only, while the right image is a typical result with dual recycling. In this latter case the beam shape is very close to the profile of a fundamental Gaussian mode (with most of the power in Schnupp modulation sidebands).

our experiment. Note, further, that the light power at the photodetector includes the modulation sidebands used for phase detection. This contribution must be appropriately taken into account to obtain the "effective contrast" that indicates the quality of interference.

In practice, the contrast could not be measured directly, but was deduced from observed data. The contrast of the interferometer without the signal recycling mirror was measured to be no better than 1:1000, even with optimum alignment. This includes a small contribution from the modulation sideband power, but is dominated by the power in higher order modes.

With the signal recycling mirror in place and dual recycling in operation, the contrast could easily reach 1:3700, now dominated by the modulation sidebands, their power being enhanced by a factor of $\approx 81$ due to signal recycling. If we subtract the computed sideband power from the total power at the photodetector, the effective contrast of the interferometer would be $1: 10^{4}$ or even better.

The experiment described was the first demonstration of dual recycling with suspended optical components, and the results were in reasonable agreement with theoretical predictions. A significant mode-cleaning effect was observed. Dual recycling proved to be fully compatible with the autoalignment system. The complete control system used in our demonstration will form the basis of the control system for the GEO 600 interferometer.

The authors thank A. Freise and colleagues at Glasgow for their help and H. Klein, H. Emme, and A. Weidner for their excellent technical support. K. A. S. and K. D. S. acknowledge support from PPARC and the University of Glasgow; K.D.S. also acknowledges support from the Royal Society of Edinburgh through a BP/RSE fellowship.

*Email address: ghh@mpq.mpg.de

${ }^{\dagger}$ Present address: The Institute of Physical and Chemical Research (RIKEN), Optical Engineering Laboratory, Hirosawa 2-1, Wako, Saitama 351-0198, Japan.

[1] K. S. Thorne, in 300 Years of Gravitation, edited by S. W. Hawking and W. Israel (Cambridge University Press, Cambridge, 1987), pp. 330-458.

[2] A. Abramovici et al., Science 256, 325-333 (1992).

[3] C. Bradaschia et al., Nuclear Instrum. Methods Phys. Res., Sect. A 289, 518-525 (1990).

[4] K. Danzmann et al., in First Edoardo Amaldi Conference on Gravitational Wave Experiments, Frascati, 1994 (World Scientific, Singapore, 1995), pp. 100-111.

[5] K. Tsubono et al., in First Edoardo Amaldi Conference on Gravitational Wave Experiments, Frascati, 1994 (Ref. [4]), pp. 112-114.

[6] D. Blair et al., in Gravitational Wave Detection, Proceedings of the TAMA Workshop, Saitama, Japan, 1996, edited by K. Tsubono et al. (Universal Academy Press, Tokyo, 1997).

[7] R.W.P. Drever et al., in Quantum Optics, Experimental Gravitation, and Measurement Theory, edited by P. Meystre and M. O. Scully (Plenum Press, New York, 1983), pp. 503-514.

[8] D. Schnier et al., Phys. Lett. A 225, 210-216 (1997).

[9] B. J. Meers, Phys. Rev. D 38, 2317-2326 (1988).

[10] B. J. Meers, Phys. Lett. A 142, 465-470 (1989).

[11] K. A. Strain and B. J. Meers, Phys. Rev. Lett. 66, 13911394 (1991).

[12] G. Heinzel et al., Opt. Commun. (to be published).

[13] R. W.P. Drever et al., Appl. Phys. B 31, 97-105 (1983).

[14] J. Hough et al., in The Detection of Gravitational Radiation, edited by D.G. Blair (Cambridge University Press, Cambridge, 1991), pp. 329-351.

[15] L. Schnupp, contribution to the European Collaboration Meeting on Interferometric Detection of Gravitational Waves, Sorrento, 1988 (unpublished).

[16] D. Maass, Diploma-thesis, University of Hannover (1995); A. Barthel, Diploma-thesis, University of Hannover (1997); A. Freise, Diploma-thesis, University of Hannover (1998).

[17] M. B. Gray et al., Appl. Opt. (to be published).

[18] J. Mizuno et al. (to be published).

[19] B. J. Meers and K. A. Strain, Phys. Rev. D 43, 3117-3130 (1991). 